\title{
Outcomes of Patients Undergoing Early Sepsis Resuscitation for Cryptic Shock Compared with Overt Shock
}

\author{
Michael A. Puskarich, Stephen Trzeciak, Nathan I. Shapiro, Alan C. Heffner, Jeffrey A. \\ Kline, and Alan E. Jones on behalf of On behalf of the Emergency Medicine Shock \\ Research Network (EMSHOCKNET) \\ Department of Emergency Medicine, Carolinas Medical Center, Charlotte, NC (MAP, AH, JAK, \\ AEJ); Department of Emergency Medicine, University of Mississippi Medical Center, Jackson, MS \\ (AEJ); Departments of Medicine, Division of Critical Care Medicine and Emergency Medicine, \\ Cooper University Hospital, Camden, New Jersey (ST); Department of Emergency Medicine and \\ Center for Vascular Biology Research, Beth Israel Deaconess Medical Center, Boston, \\ Massachusetts (NIS)
}

\section{Abstract}

Introduction-We sought to compare the outcomes of patients with cryptic versus overt shock treated with an emergency department (ED) based early sepsis resuscitation protocol.

\begin{abstract}
Methods-Pre-planned secondary analysis of a large, multicenter ED-based randomized controlled trial of early sepsis resuscitation. All subjects were treated with a quantitative resuscitation protocol in the ED targeting 3 physiological variables: central venous pressure, mean arterial pressure and either central venous oxygen saturation or lactate clearance. The study protocol was continued until all endpoints were achieved or a maximum of 6 hours. Outcomes data of patients who were enrolled with a lactate $\geq 4 \mathrm{mmol} / \mathrm{L}$ and normotension (cryptic shock) were compared to those enrolled with sustained hypotension after fluid challenge (overt shock). The primary outcome was in-hospital mortality.
\end{abstract}

Results-A total of 300 subjects were enrolled, 53 in the cryptic shock group and 247 in the overt shock group. The demographics and baseline characteristics were similar between the groups. The primary endpoint of in-hospital mortality was observed in 11/53 (21\%, 95\% CI 11 to $34)$ in the cryptic shock group and $48 / 247$ (19\%, 95\% CI 15 to 25$)$ in the overt shock group, difference of $1 \%(95 \% \mathrm{CI}-10$ to 14 ; log rank test $\mathrm{p}=0.81)$.

Conclusion-Severe sepsis with cryptic shock carries a mortality rate not significantly different from that of overt septic shock. These data suggest the need for early aggressive screening for and treatment of patients with an elevated serum lactate in the absence of hypotension.

(C) 2011 Elsevier Ireland Ltd. All rights reserved.

Address for correspondence: Alan E. Jones, MD, Director of Research, Department of Emergency Medicine, University of Mississippi Medical Center, 2500 N State Street, Jackson, MS 39216, aejones@umc.edu.

Publisher's Disclaimer: This is a PDF file of an unedited manuscript that has been accepted for publication. As a service to our customers we are providing this early version of the manuscript. The manuscript will undergo copyediting, typesetting, and review of the resulting proof before it is published in its final citable form. Please note that during the production process errors may be discovered which could affect the content, and all legal disclaimers that apply to the journal pertain.

Conflict of Interest Statement

None of the authors have any conflicts of interests to report. 


\section{Introduction}

Severe sepsis hospitalizations have doubled over the last decade resulting in at least 750,000 persons affected annually in the United States (US). ${ }^{1,2}$ Estimates suggest that 500,000 patients with severe sepsis are treated annually in US emergency departments (ED). ${ }^{3}$ The Surviving Sepsis Campaign international consensus guidelines recommend protocol-driven treatment that uses quantitative resuscitation for ED patients with septic shock, underscoring the importance of early identification and treatment of these patients. ${ }^{4}$ Current consensus definition of septic shock requires suspicion of infection in the setting of either hypotension after fluid challenge or vasopressor requirement. However, some patients manifest global tissue hypoxia, evidenced by an elevated blood lactate $\geq 4 \mathrm{mmol} / \mathrm{L}$ in the setting of normotension, a state sometimes referred to as cryptic shock. ${ }^{5-7}$

Although elevated blood lactate has been previously shown to be a strong predictor of mortality in various critical care populations ${ }^{8-10}$, we are aware of no study to date that has directly compared the outcomes of patients with severe sepsis who are treated with early quantitative resuscitation for cryptic shock versus overt shock. In this study we sought to compare outcomes of consecutive, prospectively collected patients presenting to three US EDs with severe sepsis and treated with an early quantitative resuscitation protocol for cryptic shock versus overt shock.

\section{Methods}

Study Design

We conducted a preplanned secondary analysis of a recently completed prospective, parallel group, non-blinded randomized clinical trial designed to assess the non-inferiority of lactate clearance versus central venous oxygen saturation $(\mathrm{ScvO} 2)$ as the protocol endpoint that evaluated the adequacy of oxygen delivery during ED based early quantitative resuscitation of sepsis. ${ }^{11}$ The trial was registered on Clinicatrials.gov identifier NCT00372502.

The trial took place from January 2007 to January 2009 at Carolinas Medical Center, Charlotte, NC, Beth Israel Deaconess Medical Center, Boston, MA, and Cooper University Hospital, Camden, NJ, all of which are large, urban, tertiary care hospitals staffed by emergency medicine resident physicians supervised by board certified emergency medicine attending physicians. The study was approved by the Institutional Review Board at each institution (090602A) and all participants or their surrogate provided written informed consent for participation.

Consecutive patients presenting to one of the participating EDs with severe sepsis or septic shock were eligible for enrollment if they were older than 17 years, had confirmed or suspected infection, two or more systemic inflammatory response criteria ${ }^{12}$, and hypoperfusion evidenced by either a systolic blood pressure (SBP) lower than $90 \mathrm{mmHg}$ after a minimum of $20 \mathrm{~mL} / \mathrm{kg}$ rapid volume challenge or a blood lactate concentration of at least $4 \mathrm{mmol} / \mathrm{L}$. The criteria for exclusion from the study were pregnancy, any primary diagnosis other than sepsis, suspected requirement for immediate surgery within 6 hours of diagnosis, an absolute contraindication to chest or neck central venous catheterization, cardiopulmonary resuscitation, and advanced directive orders that would restrict the study procedure.

After enrollment patients were randomly assigned to 1 of 2 groups. Each group received structured quantitative resuscitation while in the ED (the resuscitation protocol can be found at:

http://jama.ama-assn.org/content/supp1/2010/02/18/303.8.739.DC1/ 
jwe05013_02_24_2010.pdf). The ScvO2 group (N=150) was resuscitated by sequentially providing therapy needed to meet thresholds of central venous pressure, followed by mean arterial pressure, and then $\mathrm{ScvO} 2$ as originally described by Rivers et al. ${ }^{13}$ The lactate clearance group $(\mathrm{N}=150)$ had similarly targeted thresholds in central venous pressure, followed by mean arterial pressure, and then lactate clearance (defined as a decrease in serum lactate of at least $10 \%$ over 2 hours) instead of $\mathrm{ScvO} 2$ to assess for adequate oxygen delivery. The study protocol was continued until all endpoints were achieved or a maximum of 6 hours. The published results of this study showed a $6 \%$ (95\% confidence intervals -3 to $14 \%$ ) in-hospital mortality difference between the two study groups, confirming the primary hypothesis of non-inferiority. ${ }^{14}$

\section{Data Analysis and Outcomes}

The primary outcome was in-hospital mortality. We compared outcomes data of patients who qualified for enrollment with cryptic shock, defined as a lactate $>4 \mathrm{mmol} / \mathrm{L}$ and normotension (SBP at least $90 \mathrm{~mm} \mathrm{Hg}$ ) to those that qualified with overt shock, defined as hypotension (SBP lower than $90 \mathrm{mmHg}$ ) after a minimum of $20 \mathrm{~mL} / \mathrm{kg}$ rapid volume challenge with Kaplan-Meier survival estimates and generalized Wilcoxon (Peto-Prentice) $\log$ rank test. Baseline characteristics and co-interventions were compared using chi-square or Fisher exact tests for categorical data, and t-tests or Mann-Whitney-U tests for continuous data, as appropriate. Secondary outcomes included ICU and hospital length of stay and inhospital hospital complications.

Odds ratios (ORs) were calculated to determine independent predictors of in-hospital mortality by using logistic regression with bootstrap correction for $95 \%$ confidence intervals. Six variables (age, sequential organ failure assessment (SOFA) score, initial lactate, pulmonary infection, presence of end stage renal disease, and group assignment (overt or cyptic shock)) were entered into the regression analysis. Continuous data are presented as means and standard deviations or medians and interquartile ranges. Categorical data are presented as proportions with $95 \%$ confidence intervals (CI). All statistical tests were two sided with $p<0.05$ considered significant. All data were analyzed using StatsDirect statistical software (StatsDirect 2.7.7, Cheshire, England).

\section{Results}

A total of 300 subjects were enrolled, 53 in the cryptic shock group and 247 in the overt shock group (Figure 1). Baseline demographics and baseline characteristics are shown in Table 1. Patients with diabetes mellitus, end-stage renal disease, and intra-abdominal infections were significantly more likely to present with cryptic shock. Blood cultures were positive in $115 / 300$ (38\%) of patients with $69 / 115(60 \%)$ being gram positive organisms and $46 / 115$ (40\%) being gram negative organisms. Baseline physiological and severity of illness characteristics are shown in Table 2. As expected, patients in the cryptic shock group had a significantly higher baseline SBP and a significantly higher blood lactate concentration.

There were no differences in co-interventions administered between the cryptic and overt shock groups (Table 3). A total of 43\% (23/53) of the patients in the cryptic shock group and $75 \%$ (184/247) of patients in the overt shock group required continuous vasopressor infusion at some point during the hospitalization. There was an equal proportion of subjects randomized to the interventional (lactate clearance) arm in both the cryptic shock (27/53, $51 \%)$ and overt shock $(123 / 247,50 \%)$ groups and there were no differences in the resuscitation goals achieved between the groups (Table 4).

The primary outcome of in-hospital mortality was observed in 11/53 (21\%, 95\% CI 11 to 34 ) of patients in the cryptic shock group compared with $48 / 247$ (19\%, 95\% CI 15 to 25$)$ in 
the overt shock group, difference of $1 \%$ (95\% CI -10 to 14). Figure 2 shows the KaplanMeier survival curve for the two groups. There was no significant difference in survival between the groups, $\log$ rank test $p=0.81$. Additionally, we found no difference in ICU or hospital length of stay or complications between the groups (Table 5). The adjusted multiple logistic regression analysis results confirmed SOFA score as the only independent predictor of mortality (OR 1.1, 95\% confidence interval 1.0 to 1.2 ). Of note, the logistic regression results support the results of our bivariate analysis by confirming that overt shock was not an independent predictor of mortality (OR $0.5,95 \%$ confidence intervals 0.2 to 1.1 ). The model showed good fit, Hosmer-Lemeshow test $\mathrm{p}=0.76$.

\section{Discussion}

In this report we document the outcome of patients with cryptic septic shock who were treated with early quantitative resuscitation as compared to patients with overt septic shock. Our findings indicate that patients who qualify for early protocolized sepsis resuscitation with cryptic shock, defined as a lactate measurement $\geq 4 \mathrm{mmol} / \mathrm{L}$ and SBP of at least $90 \mathrm{~mm}$ $\mathrm{Hg}$, have an in-hospital mortality rate (21\%) that is not different than patients who qualify with overt shock (19\%). These data highlight the need to screen patients for signs of occult hypoperfusion, and given the high mortality rate associated with an elevated serum lactate, also suggest that patients with biochemical evidence of inadequate oxygen delivery despite normal blood pressure should be included in early sepsis resuscitation pathways.

Measurement of serum lactate is an accepted method of assessing for global tissue hypoxia, and its prognostic value in various populations has been described. ${ }^{9,15,16}$ Retrospective $^{16}$ and prospective studies ${ }^{6}$ of heterogenous ED populations with suspected infection have suggested that elevated serum lactate in the setting of normotension, or cryptic shock, carries a worse prognosis than a normal serum lactate. The present study complements these previous investigations by documenting equivalent outcomes of patients with cryptic septic shock treated with early aggressive resuscitation as compared to patients with overt septic shock. It is important to note that a variety of lactate cutoffs have been reported in the literature as abnormal, and that dichotomizing the lactate into normal or abnormal may significantly reduce the predictive value of the test. ${ }^{16}$ However, for the purposes of this study, a lactate of $>4 \mathrm{mmol} / \mathrm{L}$ was considered the threshold for cryptic shock based on the original, and most commonly used inclusion criteria for early goal-directed therapy. ${ }^{13}$

A clinician could dismiss an elevated serum lactate in the setting of hemodynamic stability as being a less acutely ill patient than one presenting with overt hypotension after volume challenge. This view may result in a tendency to withhold certain early interventions, such as early quantitative resuscitation. However, the clinical data from the present study do not support a clear distinction between these two groups and most importantly, both have the same high risk of death. We interpret these data to indicate that early, aggressive resuscitation protocols available for patients in overt shock should be strongly considered for patients in cryptic shock.

One of the strengths of our study is that both groups were treated with an early aggressive resuscitation protocol targeting physiological endpoints, which is an important difference between this study and previous cohort studies on this topic. ${ }^{6,16}$ Additionally, in a general sense enrollment in a controlled clinical trial with specific inclusion criteria results in a more homogenous patient population than might be seen in registries or observational studies. Thus our study enhances the current literature by demonstrating that patients presenting to the ED with severe sepsis who are identified as candidates for and are treated with an early quantitative resuscitation protocol, inclusion by elevation of serum lactate of at least 4.0 
$\mathrm{mmol} / \mathrm{L}$ with normotension caries an equal risk of hospital death as overt shock, despite equally aggressive therapy.

This report has several limitations that should be noted. First, although we had robust mechanisms at each center to ensure as close to a consecutive sample as was possible, it remains possible that some of the patients with elevated lactate were not enrolled. Second, this study was conducted at institutions that had established ED based quantitative resuscitation programs for sepsis prior to initiation of the study. ${ }^{17-19}$ Therefore, our results may not be generalizable to centers that do not routinely perform early quantitative resuscitation. Third, in the parent study patients were enrolled into one of two treatment protocols, which could potentially affect outcome. However, in the present analysis there were an equal proportion of patients in the cryptic shock and overt shock groups that were assigned to each treatment group and achievement of resuscitation goals were similar in both the overt and cryptic shock groups. Therefore we do not suspect significant interaction between the trial treatment and the shock group.

\section{Conclusion}

In this analysis, we document that patients presenting with cryptic septic shock have a mortality rate that is not significantly different from that of overt septic shock. These data suggest the need for early aggressive screening for and treatment of patients with evidence of global tissue hypoxia in the absence of hypotension.

\section{Acknowledgments}

This work was supported by grant K23GM076652 (Jones) from the National Institute of General Medical Sciences/ National Institutes of Health. Dr Puskarich has salary support by grant 10POST3560001 from the American Heart Association. Dr. Trzeciak is supported by grant GM083211 from the National Institute of General Medical Sciences/National Institutes of Health. Dr Shapiro was supported by grants HL091757 and GM076659 from the NIH. The sponsors had no role in the collection, analysis, or interpretation of the study data, nor in the writing of the manuscript.

\section{Reference List}

1. Angus D, Linde-Zwirble W, Lidicker J, Clermont G, Carcillo J, Pinsky M. Epidemiology of severe sepsis in the United States: analysis of incidence, outcome, and associated costs of care. Crit Care Med. 2001; 29:1303-1310. [PubMed: 11445675]

2. Dombrovskiy VY, Martin AA, Sunderram J, Paz HL. Rapid increase in hospitalization and mortality rates for severe sepsis in the United States: A trend analysis from 1993 to 2003. Crit Care Med. 2007; 35:1244-1250. [PubMed: 17414736]

3. Wang HE, Shapiro NI, Angus DC, Yealy DM. National estimates of severe sepsis in United States emergency departments. Crit Care Med. 2007; 35:1928-1936. [PubMed: 17581480]

4. Dellinger RP, Levy MM, Carlet JM, et al. Surviving sepsis campaign: International guidelines for management of severe sepsis and septic shock: 2008. Crit Care Med. 2008; 36:296-327. [PubMed: 18158437]

5. Donnino MW, Nguyen B, Jacobsen G, Tomianovich M, Rivers E. Cryptic septic shock: a subanalysis of early, goal-directed therapy. Chest. 2003; 124 90S-90b.

6. Howell MD, Donnino M, Clardy P, Talmor D, Shapiro NI. Occult hypoperfusion and mortality in patients with suspected infection. Intensive Care Med. 2007; 33:1892-1899. [PubMed: 17618418]

7. Trzeciak S. Lac-time? Crit Care Med. 2004; 32:1785-1786. [PubMed: 15286560]

8. Henning R, Weil M, Weiner F. Blood lactate as a prognostic indicator of survival in patients with acute myocardial infarction. Circ Shock. 1982; 9:307-315. [PubMed: 7094222]

9. Abramson D, Scalea T, Hitchcock R, Trooskin S, Henry S, Greenspan J. Lactate clearance and survival following injury. J Trauma. 1993; 35:584-589. [PubMed: 8411283] 
10. Shapiro NI, Howell MD, Talmor D, et al. Serum lactate as a predictor of mortality in emergency department patients with infection. Ann Emerg Med. 2005; 45:524-528. [PubMed: 15855951]

11. Jones AE, Shapiro NI, Trzeciak S, et al. Lactate clearance vs central venous oxygen saturation as goals of early sepsis therapy: a randomized clinical trial. JAMA. 2010; 303:739-746. [PubMed: 20179283]

12. Bone R, Balk R, Cerra F, et al. Definitions for sepsis and organ failure and guidelines for the use of innovative therapies in sepsis. The ACCP/SCCM Consensus Conference Committee. American College of Chest Physicians/Society of Critical Care Medicine. Chest. 1992; 101:1644-1655. [PubMed: 1303622]

13. Rivers E, Nguyen B, Havstad S, et al. Early goal-directed therapy in the treatment of severe sepsis and septic shock. N Engl J Med. 2001; 345:1368-1677. [PubMed: 11794169]

14. Jones AE, Shapiro NI, Trzeciak S, et al. Lactate clearance vs central venous oxygen saturation as goals of early sepsis therapy: a randomized clinical trial. JAMA. 2010; 303:739-746. [PubMed: 20179283]

15. Bernardin G, Pradler C, Tiger F, Deloffre P, Mattei M. Blood pressure and arterial lactate level are early indicators of short-term survival in human septic shock. Intensive Care Med. 1996; 22:1725. [PubMed: 8857433]

16. Mikkelsen M, Miltiades A, Gaieski D, et al. Serum Lactate is Associated with Mortality in Severe Sepsis Independent of Organ Failure and Shock. Crit Care Med. 2009; 37:1670-1677. [PubMed: 19325467]

17. Jones AE, Focht A, Horton JM, Kline JA. Prospective external validation of the clinical effectiveness of an emergency department-based early goal directed therapy protocol for severe sepsis and septic shock. Chest. 2007; 132:425-432. [PubMed: 17573521]

18. Trzeciak S, Dellinger RP, Abata NL, et al. Translating research to clinical practice: a 1-year experience with implementing early goal-directed therapy for septic shock in the emergency department. Chest. 2006; 129:225-235. [PubMed: 16478835]

19. Shapiro NI, Howell MD, Talmor D, et al. Implementation and outcomes of the Multiple Urgent Sepsis Therapies (MUST) protocol. Crit Care Med. 2006; 34:1025-1032. [PubMed: 16484890] 


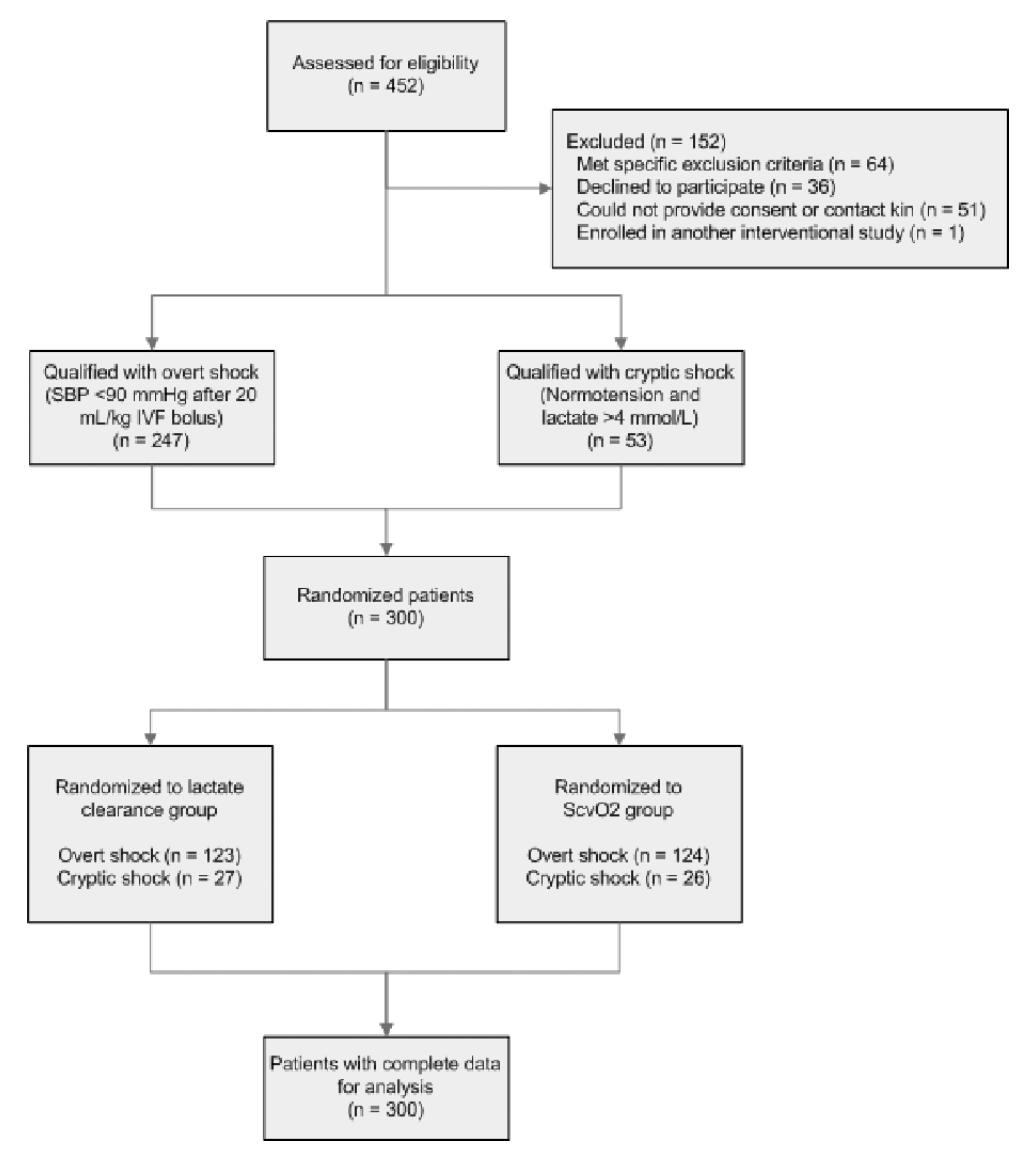

Figure 1.

Study flow diagram 


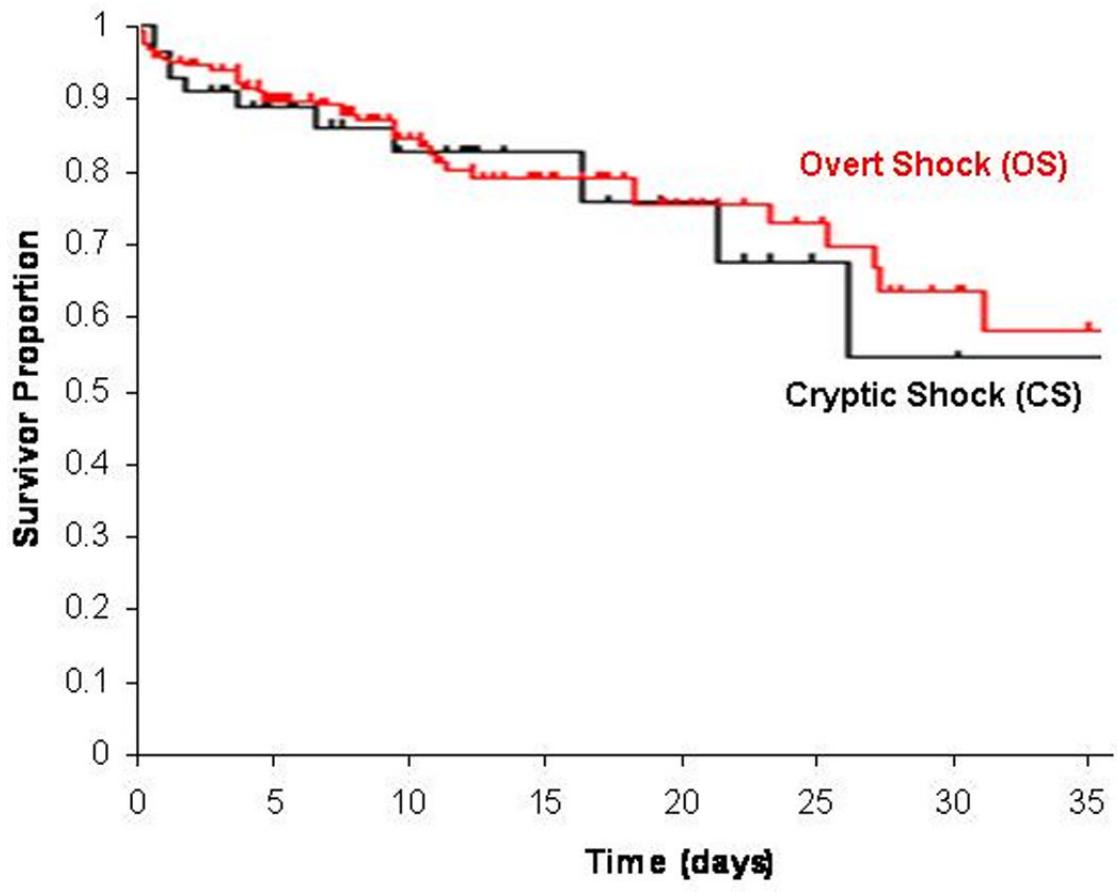

Figure 2.

Kaplan-Meier survival curves for overt shock and cryptic shock groups 
Table 1

Patient demographics and clinical characteristics.

\begin{tabular}{|c|c|c|c|}
\hline Variable & $\underset{(\mathbf{N}=53)}{\text { CS group }}$ & $\begin{array}{c}\text { OS group } \\
(\mathbf{N}=\mathbf{2 4 7})\end{array}$ & $P$ value \\
\hline Age $^{*}$ & $65(55,74)$ & $61(49,71)$ & 0.14 \\
\hline \multicolumn{4}{|l|}{ Race $(\%)$} \\
\hline Caucasian & $28(53)$ & $136(55)$ & 0.89 \\
\hline Black American & $18(34)$ & $84(34)$ & \\
\hline Other & $7(13)$ & $27(11)$ & \\
\hline \multicolumn{4}{|l|}{$\operatorname{Sex}(\%)$} \\
\hline Male & $26(49)$ & $137(55)$ & 0.49 \\
\hline Female & $27(51)$ & $110(45)$ & \\
\hline \multicolumn{4}{|l|}{ Co-morbidities (\%) } \\
\hline Diabetes mellitus & $26(49)$ & $76(31)$ & 0.02 \\
\hline Chronic obstructive pulmonary disease & $9(17)$ & $41(17)$ & 0.99 \\
\hline Human immunodeficiency virus & $2(4)$ & $23(21)$ & 0.27 \\
\hline End stage renal disease & $10(19)$ & $19(8)$ & 0.03 \\
\hline Active malignancy & $13(25)$ & $61(25)$ & 0.99 \\
\hline Organ transplant & $3(6)$ & $8(3)$ & 0.42 \\
\hline Indwelling vascular line & $5(9)$ & $38(15)$ & 0.36 \\
\hline Nursing home resident & $7(13)$ & $49(20)$ & 0.35 \\
\hline Do not resuscitate & $2(4)$ & $7(3)$ & 0.66 \\
\hline \multicolumn{4}{|l|}{ Disease severity ${ }^{*}+$} \\
\hline SAPS II score & $43(32,54)$ & $42(31,55)$ & 0.94 \\
\hline SOFA score & $6(4,9)$ & $6(4,9)$ & 0.44 \\
\hline MEDS score & $11(9,12)$ & $11(8,14)$ & 0.62 \\
\hline \multicolumn{4}{|l|}{ Suspected Source of Infection (\%) } \\
\hline Pulmonary & $10(19)$ & $92(37)$ & 0.02 \\
\hline Urinary tract & $13(25)$ & $66(27)$ & \\
\hline Intra-abdominal & $16(30)$ & $33(13)$ & \\
\hline Skin/soft tissue & $7(13)$ & $28(11)$ & \\
\hline Blood & $1(2)$ & $10(4)$ & \\
\hline Unknown & $6(11)$ & $18(7)$ & \\
\hline
\end{tabular}

Abbreviations: CS - cryptic shock; OS - overt shock; SAPS - simple acute physiology score; SOFA - Sequential Organ Failure Assessment; MEDS - mortality in emergency department sepsis

* Median (IQR)

${ }^{+}$Disease severity scores calculated at time of enrollment 
Table 2

Physiological, severity of illness, and laboratory measurements.

\begin{tabular}{lc}
\hline Variable* $^{*}$ & Initial Value \\
\hline Systolic blood pressure (mm Hg) & \\
Cryptic shock group & $108(92,126)$ \\
Overt shock group & $85(77,98)$ \\
P value & $<0.01$ \\
Heart rate (beats/min) & \\
Cryptic shock group & $114(91,128)$ \\
Overt shock group & $102(85,120)$ \\
P value & 0.04 \\
Central venous pressure (mm Hg) & \\
Cryptic shock group & $9(5,14)$ \\
Overt shock group & $10(7,14)$ \\
P value & 0.33 \\
Central venous oxygen saturation $(\%)$ & \\
Cryptic shock group & $79(65,84)$ \\
Overt shock group & $78(64,87)$ \\
P value & 0.67 \\
Lactate level (mmol/L) & \\
Cryptic shock group & \\
Overt shock group & \\
P value & \\
Respiratory Rate (breaths/min) & \\
Cryptic shock group & \\
Overt shock group & $26(15,6(13,15,15)$ \\
P value & $2.6(1.4,4.3)$ \\
Glasgow coma scale & \\
Cryptic shock group & \\
Overt shock group & \\
P value & \\
\hline Median (IQR) & \\
\hline
\end{tabular}

Abbreviations: mm Hg - millimeters of mercury; min - minutes; mmol - millimoles; L - liter 


\section{Table 3}

Administered treatments and resuscitation endpoints.

\begin{tabular}{|c|c|}
\hline Intervention & Value \\
\hline \multicolumn{2}{|c|}{ Total crystalloid volume, 0-6 hrs (L) ${ }^{*}$} \\
\hline CS group & $4.0(2.1,5.6)$ \\
\hline OS group & $4.6(2.8,6.0)$ \\
\hline$P$ value & 0.17 \\
\hline \multicolumn{2}{|c|}{ Corticosteroids, 0-6 hrs n, (\%) } \\
\hline CS group & $5(9)$ \\
\hline OS group & $39(16)$ \\
\hline$P$ value & 0.33 \\
\hline \multicolumn{2}{|c|}{ Time to initial antibiotics $(\min )^{*},+$} \\
\hline CS group & $116(70,162)$ \\
\hline OS group & $113(62,175)$ \\
\hline$P$ value & 0.63 \\
\hline \multicolumn{2}{|c|}{ Mechanical ventilation $n,(\%)$} \\
\hline CS group & $19(32)$ \\
\hline OS group & $77(31)$ \\
\hline$P$ value & 0.61 \\
\hline \multicolumn{2}{|c|}{ Activated Protein C n, (\%) } \\
\hline CS group & $1(2)$ \\
\hline OS group & $4(2)$ \\
\hline$P$ value & 0.99 \\
\hline
\end{tabular}

Abbreviations: CS, cryptic shock; OS, overt shock ; PRBC, packed red blood cell

* Median (IQR)

${ }^{+}$Time from triage to initiation of antibiotics 
Table 4

Resuscitation goals achieved.

\begin{tabular}{lccc}
\hline Goal & $\begin{array}{c}\text { CS group } \\
(\mathbf{N = 5 3})\end{array}$ & $\begin{array}{c}\text { OS group } \\
(\mathbf{N = 2 4 7})\end{array}$ & P value \\
\hline Central venous pressure $\geq \mathbf{8 ~} \mathbf{m m H g}(\boldsymbol{\%})$ & $49(92)$ & $220(89)$ & 0.46 \\
Mean arterial pressure $\geq \mathbf{6 5} \mathbf{~ m m H g ~ ( \% )}$ & $50(94)$ & $233(94)$ & 0.99 \\
$\begin{array}{l}\text { Central venous oxygen saturation } \geq \mathbf{7 0 \%}(\boldsymbol{\%}) \\
\text { or Lactate Clearance } \geq \mathbf{1 0 \% ( \% )}\end{array}$ & $51(96)$ & $237(97)$ & 0.69 \\
\hline
\end{tabular}

Abbreviations: CS - cryptic shock; OS - overt shock; mm Hg - millimeters of mercury

* Depending on protocol group assignment 
Table 5

Hospital mortality and length of stay

\begin{tabular}{lccc}
\hline Variable & $\begin{array}{c}\text { CS group } \\
(\mathbf{N = 5 3 )}\end{array}$ & $\begin{array}{c}\text { OS group } \\
(\mathbf{N = 2 4 7 )}\end{array}$ & P value \\
\hline In-hospital mortality $(\%)^{*}$ & $11(21)$ & $48(19)$ & 0.82 \\
Length of Stay ${ }^{+}$ & & & \\
$\quad$ ICU & $3.3(2,6.2)$ & $3.0(1.7,6.5)$ & 0.80 \\
$\quad$ Hospital & $8(4.7,14)$ & $8(5,13.9)$ & 0.84 \\
Hospital complications $(\%)$ & & & \\
$\quad$ Multiple organ failure & $15(28)$ & $54(22)$ & 0.41 \\
$\quad$ Care withdrawn & $9(17)$ & $28(11)$ & 0.39 \\
\hline
\end{tabular}

Abbreviations: CS - cryptic shock; OS - overt shock; ICU - intensive care unit

* Primary study endpoint

${ }^{+}$Median (IQR) 